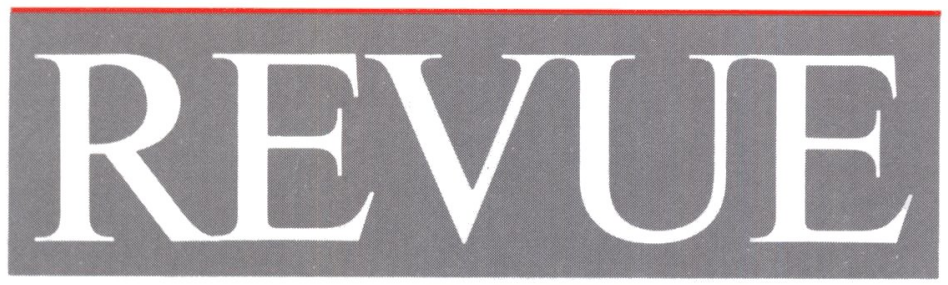

\title{
INTERNATIONALE
}

\section{DE LA CROIX-ROUGE}

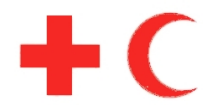

Publication bimestrielle du Comité international de la Croix-Rouge au service du Mouvement international de la Croix-Rouge et du Croissant-Rouge 


\section{COMITÉ INTERNATIONAL DE LA CROIX-ROUGE}

M. CORNELIO SOMMARUGA, docteur en droit de l'Université de Zurich, dr h.c. rer, pol. de l'Université de Fribourg (Suisse), dr h.c. en relations internationales de l'Université do Minho, Braga (Portugal), président (membre depuis 1986)

MM. MAURICE AUBERT, docteur en droit, vice-président (1979)

CLAUDIO CARATSCH, licencié ès lettres, diplômé de l'Institut d'études politiques de Paris, ancien ambassadeur, vice-prêsident dès le $1^{\text {er }}$ juin 1990 (1990)

$\mathrm{M}^{\mathrm{me}}$ DENISE BINDSCHEDLER-ROBERT, docteur en droit, professeur honoraire de l'Institut universitaire de hautes études internationales, Genève, juge à la Cour européenne des droits de l'homme, vice-présidente de 1986 à 1990 (1967)

MM. ULRICH MIDDENDORP, docteur en médecine, chef de la clinique chirurgicale de l'Hôpital cantonal, Winterthour (1973)

ATHOS GALLINO, docteur honoris causa de l'Université de Zurich, docteur en médecine, ancien maire de Bellinzone (1977)

RUDOLF JÄCKLI, docteur ès sciences (1979)

DIETRICH SCHINDLER, docteur en droit, professeur à l'Université de Zurich (1961-1973) (1980)

HANS HAUG, docteur en droit, professeur honoraire de l'Ecole des hautes études économiques, juridiques et sociales de Saint-Gall, ancien président de la Croix-Rouge suisse (1983)

PIERRE KELLER, docteur en philosophie en relations internationales (Yale), banquier (1984)

ANDRÉ GHELFI, ancien secrétaire central et vice-président de la Fédération suisse des travailleurs de la métallurgie (1985)

Mme RENÉE GUISAN, secrétaire générale de l'«Institut de la Vie» international, membre de la fondation suisse Pro Senectute, membre de l'«International Association for Volunteer Effort» (1986)

M. ALAIN B. ROSSIER, docteur en médecine, ancien professeur de rééducation des paraplégiques à l'Université de Harvard, privat-docent à la faculté de médecine de l'Université de Genève, professeur en paraplégiologie à l'Unversité de Zurich, ancien président de la Société médicale internationale de paraplégie (1986)

$M^{\text {me }}$ ANNE PETITPIERRE, docteur en droit, avocate, chargée de cours à la Faculté de droit de Genève (1987)

M. PAOLO BERNASCONI, avocat, lic. iur., chargé de cours de droit pénal économique aux Universités de Saint-Gall et de Zurich, ancien Procureur Général à Lugano, commissaire de la Fondation suisse Pro Juventute (1987)

$M^{\text {me }} \quad$ LISELOTTE KRAUS-GURNY, docteur en droit de l'Université de Zurich (1988)

$M^{\text {me }}$ SUSY BRUSCHWEILER, infirmière, directrice de l'Ecole supérieure d'enseignement infirmier de la Croix-Rouge suisse à Aarau, présidente de l'Association Suisse des directrices et directeurs d'Ecoles de Soins infirmiers (1988)

MM. JACQUES FORSTER, docteur en sciences économiques, professeur, directeur de l'Institut universitaire d'études du développement (IUED) à Genève (1988)

PIERRE LANGUETIN, licencié ès sciences économiques et sociales, docteur honoris causa de l'Université de Lausanne, ancien président de la direction générale de la Banque nationale suisse (1988)

JACQUES MOREILLON, licencié en droit, docteur ès sciences politiques, Secrétaire général de l'Organisation mondiale du Mouvement Scout, ancien Directeur général au CICR (1988)

MAX DAETWYLER, licencié en sciences économiques et sociales de l'Université de Genève, «Scholar in Residence», International Management Institute (IMI) de Genève (1989)

MARCO MUMENTHALER, docteur en médecine, professeur ordinaire de neurologie, recteur de l'Université de Berne (1989)

\section{CONSEIL EXÉCUTIF}

M. CORNELIO SOMMARUGA, président

M. MAURICE AUBERT

M. ATHOS GALLINO

M. RUDOLF JÄCKLI

M. PIERRE KELLER

M. ANDRÉ GHELFI

$M^{\text {me }}$ ANNE PETITPIERRE 


\section{REVUE INTERNATIONALE DE LA CROIX-ROUGE}

\section{SOMMAIRE}

MAI-JUIN 1990

No 783

\section{CROIX-ROUGE, CROISSANT-ROUGE ET COMMUNICATION}

\section{PROBLÉMATIQUE DE LA COMMUNICATION \\ DANS LE MONDE ACTUEL}

Sylvie Boiton-Malherbe: Les enjeux de la communication dans le monde moderne: distribution ou partage . . . . . . . . . . .

\section{UNE STRATÉGIE DE LA COMMUNICATION POUR LE MOUVEMENT INTERNATIONAL DE LA CROIX-ROUGE ET DU CROISSANT-ROUGE}

Michèle Mercier et George Reid: Une identité globale pour la Croix-Rouge et le Croissant-Rouge . . . . . . . . . . . . 208 


\section{COMMUNICATION ET PROGRAMMES D'ACTIVITÉ DES SOCIÉTÉS NATIONALES DE LA CROIX-ROUGE ET DU CROISSANT-ROUGE}

\section{Communication et diffusion}

CROIX-ROUGE PHILIPPINE

Edilberto H. Angco: Promouvoir la diffusion: rôle de la communication

CROISSANT-ROUGE MAROCAIN

Ben Saoud Badreddine: Aspects pratiques de l'information et de la diffusion . . . . . . . . . . . . . . . . . . .

\section{Communication et services communautaires}

\section{CROIX-ROUGE DU MALAWI}

Grace Paliani: La communication, moyen de promotion des services à la communauté . . . . . . . . . . . . . . . . . . .

\section{Communication, recrutement de membres} et recherche de fonds

CROIX-ROUGE NÉERLANDAISE

Marcel P.L. Vergeer: Communication et collecte de fonds pour les activités humanitaires $\ldots \ldots \ldots \ldots \ldots \ldots \ldots \ldots$

4. Communication et grand public

CROIX-ROUGE AMERICAINE

Ann Stingle et Bud Good: Communication optimale et grand public .

5. La Croix-Rouge, le Croissant-Rouge et les médias CROIX-ROUGE D'EL SALVADOR

Services d'information et médias: un dialogue permanent . . . . . . .

CROIX-ROUGE BULGARE

Encho Gospodinov: Relations avec les médias: une coopération aux multiples facettes ...................... 266 


\section{PROGRAMME DE DÉVELOPPEMENT EN MATIÈRE D'INFORMATION}

\section{CROIX-ROUGE FINLANDAISE}

Helena Korhonen: Soutien aux services d'information des Sociétés nationales en développement $\ldots \ldots \ldots \ldots \ldots \ldots \ldots \ldots$

\section{COMITÉ INTERNATIONAL DE LA CROIX-ROUGE}

Sous le signe de la "mobilisation humanitaire» — Le président du

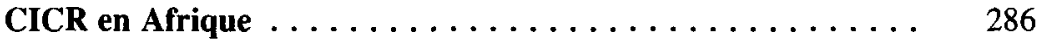

Séminaire de chirurgie de guerre (Genève, 30 mars-1 ${ }^{\text {er }}$ avril 1990) . . 290 ACTIVITÉS EXTÉRIEURES:

Afrique - Amérique latine - Asie - Moyen-Orient - Europe . .

\section{DANS LE MONDE DE LA CROIX-ROUGE ET DU CROISSANT-ROUGE}

Journée mondiale 1990 de la Croix-Rouge et du Croissant-Rouge . $\quad 300$

Réunions statutaires à Genève (30 avril-4 mai 1990) . . . . . . . 302

\section{FAITS ET DOCUMENTS}

Deuxième Colloque régional sur le droit international humanitaire (Kinshasa, 14-17 mars 1990) . . . . . . . . . . . . . . 305

La République arabe du Yémen ratifie les Protocoles . . . . . . . 307

Décès du professeur Paul Reuter . . . . . . . . . . . . 307

\section{LIVRES ET REVUES}

La protection des journalistes en mission périlleuse dans les zones de conflit armé (Sylvie Boiton-Malherbe) . . . . . . . . . . . 309

Adresses des Sociétés nationales de la Croix-Rouge et du Croissant-Rouge .................... 313 